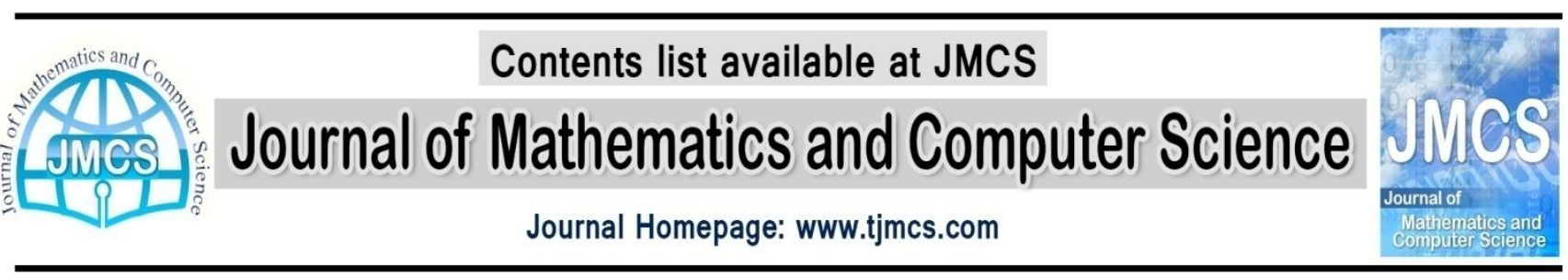

\title{
A Note on $\boldsymbol{t}$-Derivations of $\boldsymbol{B}$-Algebras
}

\author{
Rasoul Soleimani ${ }^{1}$ \\ Department of Mathematics, Payame Noor University, I.R of IRAN \\ r_soleimani@pnu.ac.ir \& rsoleimanii@yahoo.com \\ Somayeh Jahangiri \\ Department of Mathematics, Payame Noor University, I.R of IRAN \\ sjahan1389@gmail.com
}

Article history:

Received February 2014

Accepted March 2014

Available online April 2014

\section{Abstract}

In this paper, we introduce the notion of $t$-derivation on $B$-algebras and obtain some of its related properties.

Keywords: $B$-algebra, 0 -commutative, $t$-derivation.

2010 Mathematics Subject Classification: Primary 06F35, 03G25.

\section{Introduction}

Imai and Is'eki [4, 5] introduced two classes of logical algebras: $B C K$ and $B C I$-algebras. It is known that the class of $B C K$-algebras is a proper subclass of the class of $B C I$-algebras. Neggers and Kim [7] introduced the notion of $B$-algebras which is related to several classes of algebras such as $B C I / B C K$ algebras. Abujabal and Al-Shehrie [1] defined and studied the notion of left derivation of $B C I$ algebras. Further, Al-Shehrie [2] has applied the notion of left-right derivation in $B C I$-algebra to $B$ algebra and obtained some of its properties. Furthermore This logical algebra Have been studied by another authors, see for example [3], [6], [8], [9]. In this paper, we introduce the notion of $t$ derivation on $B$-algebras and investigate some properties of 0 -commutative $B$-algebras.

(see [2], [3], [6], [7], [9]) A $B$-algebra is a non-empty set $X$ with a constant 0 and a binary operation " * " satisfying the following axioms:

\footnotetext{
${ }^{1}$ This research was in part supported by a grant from Payame Noor University
} 
$(B 1) x * x=0$

(B2) $x * 0=x$;

(B3) $(x * y) * z=x *(z *(0 * y))$ for all $x, y, z \in X$.

In any $B$-algebra $X$ the following properties satisfied for all $x, y, z \in X$,

(1) $(x * y) *(0 * y)=x$.

(2) $x * y=z * y$ implies $x=z$.

(3) $x *(y * z)=(x *(0 * z)) * y$.

(4) $x * y=0$ implies $x=y$.

(5) $x=0 *(0 * x)$.

(6) $x * y=x * z$ implies $y=z$.

(7) $0 *(x * y)=y * x$.

(8) $(x * y) *(z * y)=x * z$.

A $B$-algebra $(X, *, 0)$ is said to be 0 -commutative if $x *(0 * y)=y *(0 * x)$, for any $x, y \in X$.

For any 0 -commutative $B$-algebra $X$ and all $x, y, z, u \in X$, the following properties hold:

(9) $(0 * x) *(0 * y)=y * x$.

(10) $(x * y) *(z * u)=(u * y) *(z * x)$.

(11) $(x * y) *(x * z)=z * y$.

(12) $(x * y) * z=(0 * y) *(z * x)$.

(13) $x *(y * z)=z *(y * x)$.

(14) $(x * y) * z=(x * z) * y$.

(15) $x *(x * y)=y$.

Let $X$ be a $B$-algebra. Then $X$ is called associative if for all $x, y, z \in X$,

(16) $(x * y) * z=x *(y * z)$.

For a $B$-algebra $X$, we denote $x \Lambda y=y *(y * x)$ for all $x, y \in X$.

\section{2. t-Derivation of B-Algebras}

In this section we investigate the notion of $t$-derivation for a $B$-algebra and study some of its properties.

Definition 2.1. Let $X$ be a $B$-algebra. For any $t \in X$, we define a self map $d_{t}: X \rightarrow X$ by $d_{t}(x)=x * t$ for all $x \in X$.

Lemma 2.2. Let $d_{t}$ be a self map of a $B$-algebra $X$. Then the following hold:

(i) $d_{t}$ is one-one.

(ii) $d_{t}(x) * d_{t}(y)=x * y$ for all $x, y \in X$.

Proof. It is sufficient to prove (ii). By applying (8) we obtain 


$$
d_{t}(x) * d_{t}(y)=(x * t) *(y * t)=x * y .
$$

Definition 2.3. A self map $d_{t}$ of a $B$-algebra $X$ is said to be $t$-regular if $d_{t}(0)=0$.

Lemma 2.4. Let $d_{t}$ be a self map of a 0 -commutative $B$-algebra $X$. Then the following hold:

(i) $d_{t}(x * y)=d_{t}(x) * y$ for all $x, y \in X$.

(ii) If $d_{t}$ is $t$-regular, then it is an identity.

Proof. ( $i$ ) Since $d_{t}$ is a self map of a B-algebra $X$, by (14),

$d_{t}(x * y)=(x * y) * t=(x * t) * y=d_{t}(x) * y$.

(ii) Let $d_{t}$ be $t$-regular and $x \in X$. Then $0=d_{t}(0)$ and by $(i), o=d_{t}(x) * x$. Hence by (4) $d_{t}(x)=x$ for all $x \in X$. Therefore $d_{t}$ is an identity. This completes the proof.

Definition 2.5. Let $X$ be a $B$-algebra. Then for any $t \in X$, the self map $d_{t}: X \rightarrow X$ is called a leftright $t$-derivation (or briefly $(l, r)$-t-derivation) of $X$ if it satisfies the identity $d_{t}(x * y)=\left(d_{t}(x) *\right.$ y) $\Lambda\left(x * d_{t}(y)\right)$ for all $x, y \in X$.

Similarly, if $d_{t}$ satisfies the identity $d_{t}(x * y)=\left(x * d_{t}(y)\right) \Lambda\left(d_{t}(x) * y\right)$ for all $x, y \in X$, then it is called a right-left $t$-derivation (or briefly $(r, l)$-t-derivation) of $X$.

Moreover, if $d_{t}$ is both a $(l, r)$ - and a $(r, l)$-t-derivation of $X$, then $d_{t}$ is a $t$-derivation of $X$.

Example 2.6. Let $X$ be a $B$-algebra of all real numbers except for a negative integer $-n$, with a binary operation $*$ on $X$ by $x * y=\frac{n(x-y)}{n+y}$.

For any $t \epsilon X$, define a self map $d_{t}: X \rightarrow X$ by $d_{t}(x)=x * t$ for all $x \in X$. First, we show that $X$ is a 0 -commutative $B$-algebra. For any $x, y \in X$ :

$x *(0 * y)=x * \frac{n(0-y)}{n+y}=x * \frac{-n y}{n+y}=\frac{n x+x y+n y}{n}$.

Also, $y *(0 * x)=y * \frac{n(0-x)}{n+x}=y * \frac{-n x}{n+x}=\frac{n y+y x+n x}{n}$.

Hence $X$ is a 0 -commutative $B$-algebra. Next for all $x, y, t \in X$,

$$
(x * y) * t=n \frac{n(x-y)-t(n+y)}{(n+y)(n+t)} \text {, and }(x * t) * y=n \frac{n(x-y)-t(n+y)}{(n+y)(n+t)} \text {. }
$$

Since $X$ is a 0 -commutative $B$-algebra, by (15) for all $x, y, t \in X$,

$$
\begin{aligned}
& \left(d_{t}(x) * y\right) \Lambda\left(x * d_{t}(y)\right)=(x *(y * t)) *((x *(y * t)) *((x * t) * y)) \\
& =(x * t) * y=n \frac{n(x-y)-t(n+y)}{(n+y)(n+t)}=(x * y) * t=d_{t}(x * y) .
\end{aligned}
$$

So $d_{t}$ is a $(l, r)$-t-derivation of $X$. It is easy to check that $d_{t}$ is not a $(r, l)$-t-derivation of $X$.

Example 2.7 Let $X:=\{0,1,2\}$ be a $B$-algebra with the following table,

\begin{tabular}{|r|r|r|r|}
\hline$*$ & 0 & 1 & 2 \\
\hline 0 & 0 & 2 & 1 \\
\hline 1 & 1 & 0 & 2 \\
\hline 2 & 2 & 1 & 0 \\
\hline
\end{tabular}


For any $t \in X$, define a self map $d_{t}: X \rightarrow X$ by $d_{t}(x)=x * t$ for all $x \in X$. Then it is easy to check that $d_{t}$ is a $(l, r)$-t-derivation of $X$, which is not a $(r, l)$-t-derivation of $X$. If we set $x:=0, y:=2$ and $t:=1$, then

$d_{t}(x * y)=(x * y) * t=0 \neq 2=((x * t) * y) *(((x * t) * y) *(x *(y * t)))$

$=\left(x * d_{t}(y)\right) \Lambda\left(d_{t}(x) * y\right)$.

But if for any $t \in X$, define a self map $d_{t}: X \rightarrow X$ by $d_{t}(x)=x * t=x$

then $X$ is a $t$-derivation of $X$, which is $t$-regular.

Theorem 2.8. Let $d_{t}$ be a self map of a $B$-algebra $X$. Then

(i) If $d_{t}$ is a $(l, r)$-t-derivation and $t$-regular of $X$, then $d_{t}(x)=d_{t}(x) \Lambda x$ for all $x \in X$.

(ii) If $d_{t}$ is a $(r, l)$-t-derivation of $X$, then $d_{t}(x)=x \Lambda d_{t}(x)$ for all $x \in X$ if and only if $d_{t}$ is $t$ regular.

Proof. ( $i$ ) If $d_{t}$ is a $(l, r)$-t-derivation and $t$-regular of $X$, then by (B2)

$d_{t}(x)=d_{t}(x * 0)=\left(d_{t}(x) * 0\right) \Lambda\left(x * d_{t}(0)\right)=d_{t}(x) \Lambda(x * 0)=d_{t}(x) \Lambda x$.

(ii) Let $d_{t}$ be a $(r, l)$-t-derivation of $X$. If $d_{t}$ is $t$-regular, then by (B2)

$d_{t}(x)=d_{t}(x * 0)=\left(x * d_{t}(0)\right) \Lambda\left(d_{t}(x) * 0\right)=(x * 0) \Lambda d_{t}(x)=x \Lambda d_{t}(x)$.

Conversely, suppose that $d_{t}(x)=x \Lambda d_{t}(x)$ for all $x, y \in X$, then

$d_{t}(0)=0 \Lambda d_{t}(0)=d_{t}(0) *\left(d_{t}(0) * 0\right)=d_{t}(0) * d_{t}(0)=0$.

So $d_{t}$ is $t$-regular.

\section{3. $t$-Derivation of 0 -Commutative $B$-Algebras}

In this section, we investigate the notion of $t$-derivation for 0 -commutative $B$-algebras.

Theorem 3.1. Let $d_{t}$ be a self map of an associative 0 -commutative $B$-algebra $X$. Then $d_{t}$ is a $t$ derivation of $X$.

Proof. Since $X$ is an associative 0 -commutative $B$-algebra, we have

$d_{t}(x * y)=(x * y) * t$

$\begin{array}{ll}=(x *(y * t)) * 0 & \text { [by (B2)] }\end{array}$

$\begin{array}{rll}=(x *(y * t)) *((x *(y * t)) *(x *(y * t))) & \text { [by }(B 1)]\end{array}$

$=(x *(y * t)) *((x *(y * t)) *((x * y) * t)) \quad$ [by (16) ]

$\begin{aligned}=(x *(y * t)) *((x *(y * t)) *((x * t) * y)) & {[\text { by (14)] }}\end{aligned}$

$=((x * t) * y) \Lambda(x *(y * t))$

$=\left(d_{t}(x) * y\right) \Lambda\left(x * d_{t}(y)\right)$.

Again,

$d_{t}(x * y)=(x * y) * t=((x * t) * y) * 0$

[by (14) and (B2)]

$=((x * t) * y) *(((x * t) * y) *((x * t) * y))$

[by (B1)] 


$$
\begin{array}{llll}
=((x * t) * y) *(((x * t) * y) *((x * y) * t)) & \text { [by (14)] } \\
=((x * t) * y) *(((x * t) * y) *(x *(y * t))) & & \text { [by (16)] } \\
=(x *(y * t)) \Lambda((x * t) * y)=\left(x * d_{t}(y)\right) \Lambda\left(d_{t}(x) * y\right) . &
\end{array}
$$

Lemma 3.2. Let $d_{t}$ be a $(r, l)$-t-derivation of a 0 -commutative $B$-algebra $X$. Then $d_{t}(x * y)=x * d_{t}(y)$ for all $x, y \in X$.

Proof. Since $d_{t}$ is a $(r, l)$-t-derivation of $X$, by $(15)$,

$d_{t}(x * y)=\left(x * d_{t}(y)\right) \Lambda\left(d_{t}(x) * y\right)=\left(d_{t}(x) * y\right) *\left(\left(d_{t}(x) * y\right) *\left(x * d_{t}(y)\right)\right)$ $=x * d_{t}(y)$.

Definition 3.3. Let $X$ be a $B$-algebra and $d_{t}, d_{t}^{\prime}$ be two self maps of $X$. Then we define $d_{t} \circ d_{t}^{\prime}$ : $X \rightarrow X$ by $\left(d_{t} \circ d_{t}^{\prime}\right)(x)=d_{t}\left(d_{t}^{\prime}(x)\right)$, for all $x \in X$.

Theorem 3.4. Let $X$ be a 0 -commutative $B$-algebra and $d_{t}, d_{t}^{\prime}$ are $(r, l)$-t-derivations of $X$. Then $d_{t} \circ d_{t}^{\prime}$ is a $t$-derivation of $X$.

Proof. Since $d_{t}, d_{t}^{\prime}$ are two self maps of $X$, by Lemma 2.4(i) and (15) for all $x, y \in X$,

$$
\begin{aligned}
& \left(d_{t} \circ d_{t}^{\prime}\right)(x * y)=d_{t}\left(d_{t}^{\prime}(x * y)\right)=d_{t}\left(d_{t}^{\prime}(x) * y\right)=d_{t}\left(d_{t}^{\prime}(x)\right) * y= \\
& \quad\left(x * d_{t}\left(d_{t}^{\prime}(y)\right)\right) *\left(x * d_{t}\left(d_{t}^{\prime}(y)\right)\right) *\left(d_{t}\left(d_{t}^{\prime}(x)\right) * y\right)=\left(d_{t}\left(d_{t}^{\prime}(x)\right) * y\right) \Lambda\left(x * d_{t}\left(d_{t}^{\prime}(y)\right)\right) \\
& \quad=\left(\left(d_{t} \circ d_{t}^{\prime}\right)(x) * y\right) \Lambda\left(x *\left(d_{t} \circ d_{t}^{\prime}\right)(y)\right) .
\end{aligned}
$$

Next, since $d_{t}, d_{t}^{\prime}$ are $(r, l)$-t-derivations of $X$, by Lemma 3.2 and (15), for all $x, y \in X$,

we have

$$
\begin{gathered}
\left(d_{t} \circ d_{t}^{\prime}\right)(x * y)=d_{t}\left(d_{t}^{\prime}(x * y)\right)=d_{t}\left(x * d_{t}^{\prime}(y)\right)=x * d_{t}\left(d_{t}^{\prime}(y)\right) \\
=\left(d_{t}\left(d_{t}^{\prime}(x)\right) * y\right) *\left(\left(d_{t}\left(d_{t}^{\prime}(x)\right) * y\right) *\left(x * d_{t}\left(d_{t}^{\prime}(y)\right)\right)\right)= \\
\left(x * d_{t}\left(d_{t}^{\prime}(y)\right)\right) \Lambda\left(d_{t}\left(d_{t}^{\prime}(x)\right) * y\right)=\left(x *\left(d_{t} \circ d_{t}^{\prime}\right)(y)\right) \Lambda\left(\left(d_{t} \circ d_{t}^{\prime}\right)(x) * y\right) .
\end{gathered}
$$

Theorem 3.5. Let $X$ be a 0 -commutative $B$-algebra and let $d_{t}$ be a $(r, l)$-t-derivation and $d_{t}^{\prime}$ be a self map of $X$. Then $d_{t} \circ d_{t}^{\prime}=d_{t}^{\prime} \circ d_{t}$

Proof. Suppose $d_{t}$ is a $(r, l)$-t-derivation and $d_{t}^{\prime}$ is a self map of $X$. By Lemmas $2.4(i)$ and 3.2,

for all $x, y \in X,\left(d_{t} \circ d_{t}^{\prime}\right)(x * y)=d_{t}\left(d_{t}^{\prime}(x * y)\right)=d_{t}\left(d_{t}^{\prime}(x) * y\right)=d_{t}^{\prime}(x) * d_{t}(y)$.

Again, by Lemmas 3.2 and 2.4(i), for all $x, y \in X$,

$\left(d_{t}^{\prime} \circ d_{t}\right)(x * y)=d_{t}^{\prime}\left(d_{t}(x * y)\right)=d_{t}^{\prime}\left(x * d_{t}(y)\right)=d_{t}^{\prime}(x) * d_{t}(y)$.

Therefore, $\left(d_{t} \circ d_{t}^{\prime}\right)(x * y)=\left(d_{t}^{\prime} \circ d_{t}\right)(x * y)$

By putting $y:=0$, for all $x \in X$, we get

$\left(d_{t} \circ d_{t}^{\prime}\right)(x)=\left(d_{t}^{\prime} \circ d_{t}\right)(x)$. Hence, $d_{t} \circ d_{t}^{\prime}=d_{t}^{\prime} \circ d_{t}$.

This completes the proof.

Definition 3.6. Let $X$ be a $B$-algebra and let $d_{t}$ and $d_{t}^{\prime}$ be two self maps of $X$. Then we define $d_{t} * d_{t}^{\prime}: X \rightarrow X$ by $\left(d_{t} * d_{t}^{\prime}\right)(x)=d_{t}(x) * d_{t}^{\prime}(x)$ for all $x \in X$.

Theorem 3.7. Let $d_{t}, d_{t}^{\prime}$ be two $(r, l)$-t-derivations of a 0 -commutative $B$-algebra $X$. Then $d_{t} * d_{t}^{\prime}=d_{t}^{\prime} * d_{t}$. 
Proof. Since $d_{t}$ is a $(r, l)$-t-derivation of $X$, for all $x, y \in X$, by Lemmas 2.4(i) and 3.2,

$\left(d_{t} \circ d_{t}^{\prime}\right)(x * y)=d_{t}\left(d_{t}^{\prime}(x * y)\right)=d_{t}\left(d_{t}^{\prime}(x) * y\right)=d_{t}^{\prime}(x) * d_{t}(y)$.

Again, since $d_{t}^{\prime}$ is a $(r, l)$-t-derivation of $X$, then by Lemmas 3.2 and $2.4(i)$,

$\left(d_{t} \circ d_{t}^{\prime}\right)(x * y)=d_{t}\left(d_{t}^{\prime}(x * y)\right)=d_{t}\left(x * d_{t}^{\prime}(y)\right)=d_{t}(x) * d_{t}^{\prime}(y)$.

Therefore, $d_{t}^{\prime}(x) * d_{t}(y)=d_{t}(x) * d_{t}^{\prime}(y)$. By putting $y:=x$, for all $x \in X$, we get $d_{t}^{\prime}(x) * d_{t}(x)=d_{t}(x) * d_{t}^{\prime}(x)$. Hence $d_{t} * d_{t}^{\prime}=d_{t}^{\prime} * d_{t}$. This proves the theorem.

\section{References}

[1] H.A.S. Abujabal, N.O. Al-Shehrie, On left Derivations of $B C I$-algebras, Soochow J. Math., 33(3) (2007), 435-444.

[2] N.O. Al-Shehrie, Derivations of $B$-algebras, JKAU: Sci., 22(1) (2010), 71-83.

[3] J.R. Cho, H.S. Kim, On B-algebras and quasigroups, Quasigroups and Related Systems, 8 (2001), 16.

[4] Y.Imai, K. Is'eki, On axiom systems of propositional calculi. XIV, Proc. Japan Acad., 42(1966), 1922.

[5] K. Is'eki, An algebra related with a propositional calculus, Proc. Japan Acad., 42(1966), 26-29.

[6] H.S. Kim, H.G. Park, On 0-commutative $B$-algebras, Sci. Math. Japonicae Online, (2005), 31-36.

[7] J. Neggers, H.S. Kim, On B-algebras, Mate. Vesnik, 54 (2002), 21-29.

[8] S.A. Nematoalah Zadeh, A. Radfar, A. Borumand Saied, On $B P$-algebras and $Q S$-algebras, TJMCS, 5(1) (2012), 17-21.

[9] A. Walendziak, Some axiomatizations of $B$-algebras, Math. Slovaca, 56(3) (2006), 301-306. 